\title{
Strong IR cancellation in heavy quarkonium and precise top mass determination
}

\author{
Y. Kiyo, ${ }^{a}$ G. Mishima ${ }^{b}$ and $Y$. Sumino ${ }^{c}$ \\ ${ }^{a}$ Department of Physics, Juntendo University, \\ Inzai, Chiba 270-1695, Japan \\ ${ }^{b}$ Department of Physics, University of Tokyo, \\ Bunkyo-ku, Tokyo 113-0033, Japan \\ ${ }^{c}$ Department of Physics, Tohoku University, \\ Sendai, 980-8578 Japan \\ E-mail: ykiyo@juntendo.ac.jp, mishima@hep-th.phys.s.u-tokyo.ac.jp, \\ sumino@tuhep.phys.tohoku.ac.jp
}

ABSTRACT: Combining recent perturbative analyses on the static QCD potential and the quark pole mass, we find that, for the heavy quarkonium states $c \bar{c}, b \bar{b}$ and $t \bar{t}$, (1) ultra-soft (US) corrections in the binding energies are small, and (2) there is a stronger cancellation of IR contributions than what has been predicted by renormalon dominance hypothesis. By contrast, for a hypothetical heavy quarkonium system with a small number of active quark flavors $\left(n_{l} \approx 0\right)$, we observe evidence that renormalon dominance holds accurately and that non-negligible contributions from US corrections exist. In addition, we examine contributions of renormalons at $u=-1$. As an important consequence, we improve on a previous prediction for possible achievable accuracy of top quark $\overline{\mathrm{MS}}$-mass measurement at a future linear collider and estimate that in principle $20-30 \mathrm{MeV}$ accuracy is reachable.

KeYwords: QCD Phenomenology

ARXIV EPRINT: 1506.06542 
During the past few decades, there have been significant developments in the analysis of heavy quarkonium systems using perturbative QCD. Developments in computational technology greatly advanced our understanding on the nature of quark masses and interquark forces. We anticipate that eventually these developments will deepen our understanding on the structure of perturbative QCD in more general contexts.

Recently an important step toward this direction has been achieved. A computation was completed of the four-loop relation between the quark pole mass and the mass in the modified-minimal-subtraction scheme ( $\overline{\mathrm{MS}}$ mass) [1]. This result, when combined with other known results such as the three-loop correction $\left(a_{3}\right)$ to the static QCD potential $V_{\mathrm{QCD}}(r)[2,3]$, sets our analysis at a new stage, namely, at full next-to-next-to-next-toleading order (NNNLO) in terms of short-distance quark masses. It realizes a cancellation of infra-red (IR) dynamics at this order.

In this first analysis we report what can be learned by combining existing results. In particular we compare the results of $[1,4,5]$ to make clearer the nature of the perturbative series of the heavy quarkonium energies, concerning (1) corrections from the ultra-soft (US) energy scale and (2) the renormalon dominance hypothesis. In addition, we examine contributions of an ultra-violet (UV) renormalon at $u=-1$ and discuss possible contributions of an IR renormalon at $u=+1$.

Motivations for performing such an analysis can be stated as follows. A few years ago, a convincing evidence has been presented for the existence of IR renormalons in the perturbative series of the energy of a static color source, which has an IR structure common to the quark pole mass [6]. Hence, it is among general interests how accurately the renormalon dominance picture holds for the quark pole mass. Furthermore, contributions of US corrections to the quarkonium energy have collected attention since long time [7-11]. Despite an original expectation of being dominating at IR, there have been evidences that US corrections are moderate in size from comparisons of the perturbative predictions with experimental data for the bottomonium spectrum [12-14], phenomenological potential models of heavy quarkonia [15, 16], and lattice computations of $V_{\mathrm{QCD}}(r)$ [17-19]. However, extraction of an accurate size of the US corrections still remains a challenge [20].

Important applications of this type of analysis include precise determination of the masses of the heavy quarks $c, b$ and $t$ from the energy levels of the lowest-lying heavy quarkonium states [21-25]. (For earlier works, see [26, 27] and references therein.) In this paper we apply our new understanding to a study of the possible achievable accuracy of top quark mass measurement expected at a future linear collider. Today, a precise determination of the top quark mass is highly demanded, for a precision test of the standard model of particle physics (SM) [28-30], and also since the top quark mass plays a crucial role in the vacuum stability of the SM at a very high energy scale [31,32]. Hence, progress in our understanding of the heavy quarkonium states may lead to an access to deep aspects of the SM.

The pole- $\overline{\mathrm{MS}}$ mass relation can be expressed in a series expansion in the strong coupling constant as

$$
m_{\text {pole }}=\bar{m}\left[1+d_{0} \frac{\alpha_{s}(\bar{m})}{\pi}+d_{1}\left(\frac{\alpha_{s}(\bar{m})}{\pi}\right)^{2}+d_{2}\left(\frac{\alpha_{s}(\bar{m})}{\pi}\right)^{3}+d_{3}\left(\frac{\alpha_{s}(\bar{m})}{\pi}\right)^{4}+\mathcal{O}\left(\alpha_{s}^{5}\right)\right]
$$




\begin{tabular}{|c|ccccccc|}
\hline$n_{l}$ & 0 & 1 & 2 & 3 & 4 & 5 & 6 \\
\hline$d_{3}^{n_{h}=1}$ & 3556.5 & 2853.4 & 2232.9 & 1691.2 & 1224.0 & 827.4 & 497.2 \\
\hline
\end{tabular}

Table 1. Exact result of $d_{3}$ for $0 \leq n_{l} \leq 6$ in the full theory, with $n_{h}=1$ heavy quark and $n_{l}$ massless quarks. We use eq. (2) obtained by a fit of the results in [1,33, 34]. An error of \pm 21.5 is assigned to each value.

Here, $\bar{m} \equiv m_{\overline{\mathrm{MS}}}\left(m_{\overline{\mathrm{MS}}}\right)$ denotes the $\overline{\mathrm{MS}}$ mass renormalized at the $\overline{\mathrm{MS}}$ mass scale; $\alpha_{s}(\mu)=$ $\alpha_{s}^{\left(n_{l}\right)}(\mu)$ represents the strong coupling constant in the $\overline{\mathrm{MS}}$ scheme, where $n_{l}$ is the number of massless quark flavors $\left(n_{l}=3,4\right.$ and 5 for the charm, bottom and top quarks, respectively); the renormalization scale $\mu$ is set to $\bar{m}$. In most part of this paper, we use the coupling constant of the theory with $n_{l}$ flavors only as the expansion parameter. The coefficients $d_{i}$ can be obtained from the corresponding mass relations in the full theory (with $n_{h}$ heavy quarks and $n_{l}$ light quarks), respectively, by rewriting them in terms of the coupling constant of the theory with $n_{l}$ light quarks only.

Let us first summarize the results of the previous analyses, on which our analysis is based. Refs. [4, 5] estimated $d_{3}$ on the basis of different assumptions, prior to ref. [1], which accomplished the exact computation of $d_{3}$ :

- ref. [4] required stability of the perturbative prediction for $2 m_{\text {pole }}+V_{\mathrm{QCD}}(r)$ at relatively large $r$. Essentially the only assumption made is that US corrections in $V_{\mathrm{QCD}}(r)$ do not deteriorate perturbative stability (which holds up to NNLO) at NNNLO.

- ref. [5] assumed renormalon dominance in $m_{\text {pole }}$ and $V_{\mathrm{QCD}}(r)$ and estimated their contributions from the latter. Contribution of US corrections in $V_{\mathrm{QCD}}(r)$ was subtracted in this estimate. ${ }^{1}$

- The exact values of $d_{3}$ are obtained combining the results of direct perturbative computations in $[1,33,34]$.

Only the values for $n_{l}=3,4,5$ are presented explicitly in the final form in [1] (for the full theory with $n_{h}=1$ ). Since we need the values for other $n_{l}$ 's in our analysis, we derive the exact result of $d_{3}$ given as a cubic polynomial of $n_{l}$ as

$$
d_{3, \text { full theory }}^{\text {exact }}=-0.67814 n_{l}^{3}+43.396 n_{l}^{2}-745.85 n_{l}+3556.5
$$

where an error of \pm 21.5 is assigned to its value for each $n_{l}$. We determined the last two coefficients of eq. (2) by a fit using the results of [33, 34] in addition to the result of [1]. For the reader's convenience, we list the exact result of $d_{3}$ in the full theory in table 1 using this formula for $0 \leq n_{l} \leq 6$.

As already mentioned, we convert the above formula using the coupling of the theory with $n_{l}$ massless quarks only as the expansion parameter. This gives

$$
d_{3, \text { converted }}^{\text {exact }}=-0.67814 n_{l}^{3}+43.396 n_{l}^{2}-745.42 n_{l}+3551.1,
$$

with the same error \pm 21.5 . In the rest of the analysis, we use this $d_{3}$ for various $n_{l}$ 's.

\footnotetext{
${ }^{1}$ Since US corrections in $V_{\mathrm{QCD}}(r)$ do not contribute to the renormalon at $u=1 / 2$, this manipulation is justified within the renormalon dominance hypothesis.
} 


\begin{tabular}{|l|llllllc|}
\hline$n_{l}$ & 0 & 1 & 2 & 3 & 4 & 5 & 6 \\
\hline$d_{3}^{\text {est }}[4]$ & $3351(152)$ & - & - & $1668(167)$ & $1258_{-66}^{+26}$ & $897_{-175}^{+31}$ & - \\
$d_{3}^{\text {est }}[5]$ & $3562(173)$ & $2887(133)$ & $2291(98)$ & $1772(82)$ & $1324(81)$ & $945(92)$ & $629(191)$ \\
$d_{3}^{\text {exact }}[1]$ & $3551.1(21.5)$ & $2848.4(21.5)$ & $2228.4(21.5)$ & $1687.1(21.5)$ & $1220.3(21.5)$ & $824.1(21.5)$ & $494.3(21.5)$ \\
\hline
\end{tabular}

Table 2. Summary table of relevant estimates and exact results of $d_{3}$. The first line shows the estimates based on stability of the perturbative prediction for $2 m_{\text {pole }}+V_{\mathrm{QCD}}(r)$; the second line shows the estimates based on renormalon dominance hypothesis; the third line shows the exact results (converted to the values in the $n_{l}$ flavor theory).

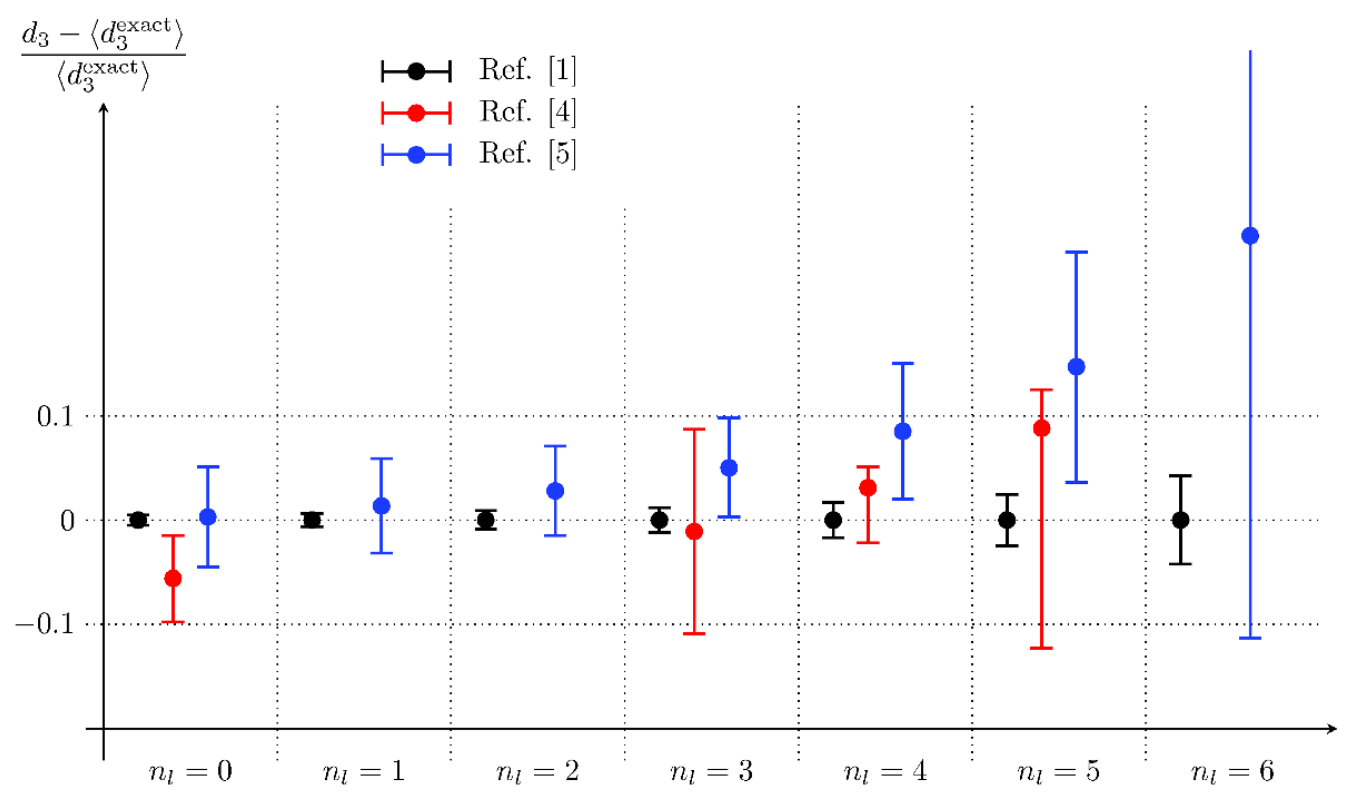

Figure 1. Comparison of $\left(d_{3}-\left\langle d_{3}^{\text {exact }}\right\rangle\right) /\left\langle d_{3}^{\text {exact }}\right\rangle$ for the (converted) exact value of $d_{3}$ and the two estimates, where $\left\langle d_{3}^{\text {exact }}\right\rangle$ denotes the central value of $d_{3}^{\text {exact }}$.

In table 2 we summarize the two estimates and the exact result for $0 \leq n_{l} \leq 6$. $^{2}$ The relative accuracies are compared visually in figure 1. Overall, we find a reasonable agreement of the previous estimates and the exact results, with respect to the assigned errors. The relative accuracies of the estimates are also fairly good, at order $10 \%$ level. These features provide certain justification to the used assumptions in these estimates.

Furthermore, we can make a closer examination. In particular, the central (optimal) values of $d_{3}^{\text {est }}$ in the table and figure carry important information on the respective assumptions. We should note that the errors of $d_{3}^{\text {est }}$ are only systematic and have no statistical nature. Hence, by carefully contemplating on the origins of these systematic errors, we can extract the sizes and signs of the systematic effects. The agreement with respect to the

\footnotetext{
${ }^{2}$ Since we use the converted $d_{3}^{\text {exact }}$, its values for $n_{l}=3,4,5$ listed in this table are different from table III of [1]. In this sense, the comparison in table III of [1] is not consistent, since $d_{3}$ 's in the different definitions are compared. Numerically the differences due to different definitions are small, nonetheless.
} 
systematic errors is a necessary condition for the validity of our analysis given below.

In the cases $n_{l}=3,4,5$, corresponding to $c \bar{c}, b \bar{b}, t \bar{t}$ quarkonium states, respectively, we see a good agreement of the estimates by [4] with the exact values, whereas the estimates by [5] are slightly larger. On the other hand, for smaller $n_{l}=0,1$, which correspond to hypothetical heavy quarkonium systems, the agreement between the estimates by [5] and the exact results is fairly good, whereas the estimate by [4] for $n_{l}=0$ is slightly smaller than the exact value. From these observations we derive the following interpretation:

- For $n_{l}=3,4,5$, (i) US corrections in $V_{\mathrm{QCD}}(r)$ are small, and (ii) there is a stronger cancellation of IR contributions than what has been predicted by renormalon dominance hypothesis.

- For $n_{l}=0$, (iii) renormalon dominance holds more accurately, and (iv) non-negligible contributions from US corrections exist.

We explain the details in the following.

The renormalon dominance hypothesis assumes that the expansion coefficient of the perturbative series is dominated by a factorial $(\sim n !)$ growth [35],

$$
d_{n} \sim \text { const. } \times\left(2 \beta_{0}\right)^{n} \frac{\Gamma(n+\nu+1)}{\Gamma(\nu+1)} \quad \text { for } \quad n \gg 1,
$$

which stems from the singularity at $u=1 / 2$ in the Borel transform of the perturbative series. [ $\nu=\beta_{1} /\left(2 \beta_{0}^{2}\right)$, and $\beta_{i}$ denotes the $(i+1)$-loop coefficient of the beta function of $\alpha_{s}$.] Contributions from the analytic part at $u=1 / 2$ are neglected. The comparison between $d_{3}^{\text {exact }}$ and the central values of $d_{3}^{\text {est }}[5]$ shows that the renormalon dominance hypothesis works better for smaller $n_{l}$. This suggests that the above factorial growth overwhelms contributions from the analytic part as $\beta_{0}(>0)$ becomes larger for smaller $n_{l}$.

Another source of $n_{l}$ dependence of the renormalon dominance resides in the series $[35,37]$

$$
\begin{aligned}
F_{n}=1 & +\frac{\nu}{n+\nu} \tilde{c}_{1}+\frac{\nu(\nu-1)}{(n+\nu)(n+\nu-1)} \tilde{c}_{2}+\frac{\nu(\nu-1)(\nu-2)}{(n+\nu)(n+\nu-1)(n+\nu-2)} \tilde{c}_{3} \\
& +\mathcal{O}\left(\frac{1}{n^{4}}\right)
\end{aligned}
$$

in eq. (33) of [5]. The factor $F_{n}$ multiplies the right-hand side of eq. (4), giving $1 / n$ suppressed corrections, so that it shows how the expansion coefficient approaches the asymptotic form at large orders $(n \gg 1) .{ }^{3}$ Figure 2 plots the series (5) in our case $n=3$ for different $n_{l}$ 's. They exhibit the tendency that $1 / n$ suppressed contributions become more important for larger $n_{l}$, although the first term $(=1)$ is by far dominating. Both of these $n_{l}$ dependences in analytic and $1 / n$ suppressed contributions have been taken into account in the error estimates of [5]. The former error enters as scale dependences in the analysis of [5] and is the main source of errors.

\footnotetext{
${ }^{3}$ Contributions from the analytic part at $u=1 / 2$ are not included in the series $F_{n}$, as they are suppressed exponentially.
} 


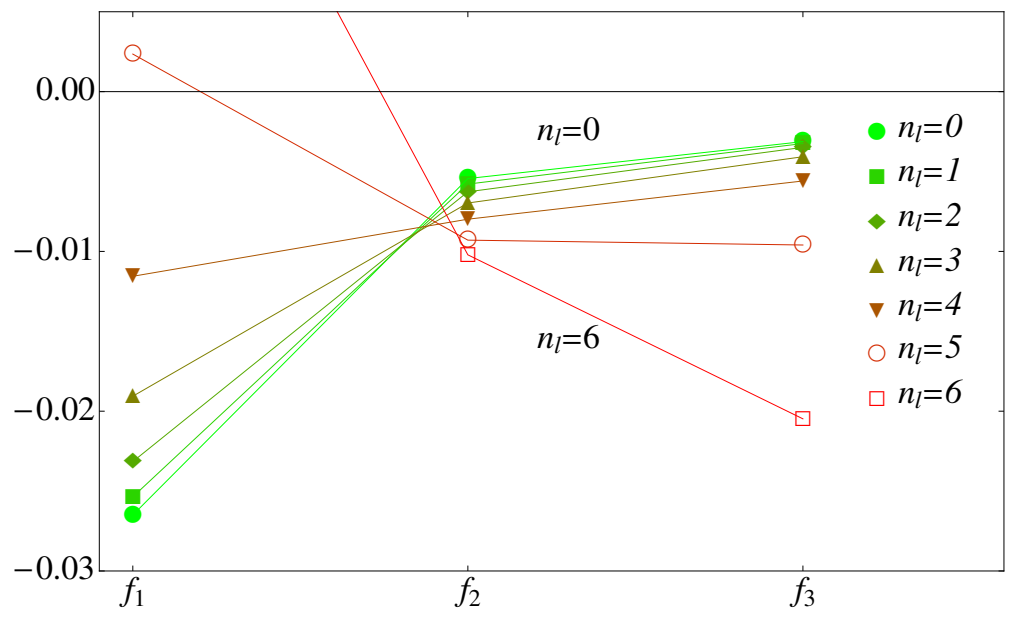

Figure 2. Each term of eq. (5) for $n=3$ and different $n_{l}$ 's: $f_{1}=\frac{\nu}{n+\nu} \tilde{c}_{1}, f_{2}=\frac{\nu(\nu-1)}{(n+\nu)(n+\nu-1)} \tilde{c}_{2}$, $f_{3}=\frac{\nu(\nu-1)(\nu-2)}{(n+\nu)(n+\nu-1)(n+\nu-2)} \tilde{c}_{3}$. The first term $\left(f_{0}=1\right)$ is omitted, since it is by far greater.

One may wonder if the UV renormalon at $u=-1$ contained in the pole mass gives a significant contribution to the perturbative series of the pole mass. Based on an analysis in the large- $\beta_{0}$ approximation, we estimate that the contribution of the $u=-1$ renormalon to $d_{3}$ is fairly small compared to the errors of $d_{3}^{\text {est }}[5]$ listed in table 2 . This is consistent, since the analytic part at $u=1 / 2$ contributes dominantly to these errors, and the $u=$ -1 renormalon belongs to the analytic part. The analysis also suggests that the $u=$ -1 renormalon contribution is not a dominant component of the analytic part. Another important feature is that, since the UV renormalon is Borel summable and gives a welldefined contribution, as long as we obtain a converging series of a physical observable (such as the heavy quarkonium energy level), the contribution of the $u=-1$ renormalon to the error estimate becomes small (arbitrarily small unlike IR renormalons). Indeed contribution to the error is minor at our present perturbative order. We give details of the analysis of the $u=-1 \mathrm{UV}$ renormalon in the appendix.

Similarly there may be effects by the $u=1$ IR renormalon contained in the pole mass, whose properties are less known. Known properties are as follows [36]. (a) It is induced by the non-relativistic kinetic energy operator $\vec{D}^{2} /(2 m)$; (b) It is not forbidden by any symmetry, and parametrically it possibly induces an order $\Lambda_{\mathrm{QCD}}^{2} / m$ uncertainty; (c) It does not appear in the large- $\beta_{0}$ approximation. With this limited knowledge, it is not easy to estimate contribution of the $u=1$ renormalon in the estimate of $d_{3}$ in [5]. In principle, this contribution is exponentially suppressed in the estimate of the $u=1 / 2$ renormalon in the pole mass and is encoded in the scale dependence in the error estimate of $d_{3}^{\text {est }}[5]$.

We turn to the estimates of $d_{3}$ by [4], which incorporate the fact that cancellation of IR dynamics occurs beyond the renormalon dominance hypothesis. It can be understood using the potential-NRQCD effective field theory [10, 11], in which interactions of a heavy quarkonium and IR degrees of freedom are systematically organized in multipole expansion 
in $r$. The leading order interaction is given by the interaction of an IR gluon with the total color charge of the heavy quarkonium, which vanishes for a color-singlet system. The corresponding contribution to the binding energy is given by an $r$-independent IR part of $2 m_{\text {pole }}+V_{\mathrm{QCD}}(r)$ [38-40]. The cancellation between $2 m_{\text {pole }}$ and $V_{\mathrm{QCD}}(r)$ is not restricted to the renormalon part, and the analytic part at $u=1 / 2$ contains such contributions.

In this general framework, the lowest order non-canceled IR contribution to the energy is given by a double insertion of the dipole interaction between the color-electric field and heavy quarkonium, expressed in terms of a non-local gluon condensate of the form $\sim\left\langle\vec{r} \cdot \vec{E}^{a} \vec{r} \cdot \vec{E}^{a}\right\rangle$. It is dominated by contributions from the US energy scale, and the perturbative evaluation of this condensate at $\mathcal{O}\left(\alpha_{s}^{4} \log \alpha_{s}\right)$ and $\mathcal{O}\left(\alpha_{s}^{4}\right)$ has been incorporated in $V_{\mathrm{QCD}}(r)$ in the estimate of $d_{3}$. In principle, the $u=1$ renormalon in the pole mass (if it exists) can affect $2 m_{\text {pole }}+V_{\mathrm{QCD}}(r)$. However, the large mass limit $m \rightarrow \infty$ is taken in the analysis of [4], so that the $u=1$ renormalon (order $\Lambda_{\mathrm{QCD}}^{2} / m$ ) is suppressed compared to the $u=3 / 2$ renormalon (order $r^{2} \Lambda_{\mathrm{QCD}}^{3}$ ). Hence, the estimate of $d_{3}^{\text {est }}$ [4] should not be affected by the $u=1$ renormalon. Furthermore, in estimating $d_{3}$ the effects of taking the large mass limit are small for $n_{l}=3,4,5$, compared to the real $c, b, t$-quark mass cases, hence, our discussion is expected to be valid for these real heavy quarkonium systems. In the cases $n_{l} \leq 2$ and $n_{l} \geq 6$, perturbative analysis makes sense only in a hypothetical static limit $m \rightarrow \infty$, and our discussion is confined to this limit.

In perturbative QCD, instability against scale variation in IR region is manifest for all the physical observables, reflecting the blow-up of the running coupling constant at IR. For a "good" observable, generally scale dependence decreases as the order of perturbative expansion is raised. Empirically this happens not only in the ultra-violet (UV) direction but also stability extends to IR region as the perturbation order is increased. In the case of the heavy quarkonium energy, the leading source of IR instability is the non-local gluon condensate dominated by US corrections. The (optimal) values of the estimates of $d_{3}$ in the first line of table 2 are chosen to optimize the stability of the perturbative prediction for the energy in the IR region at NNNLO. A very good coincidence of these values with the exact results for $n_{l}=3,4$ suggests that the US corrections are small for these systems. Here, we may set the criterion for "large" or "small" by whether the corrections deteriorate stability of the perturbative prediction or not.

As shown in [4], perturbative stability of $2 m_{\text {pole }}+V_{\mathrm{QCD}}(r)$ is sensitive to the precise value of $d_{3}$, and this sensitivity turns out to be asymmetric with respect to the sign of a variation of $d_{3} \cdot{ }^{4}$ If $d_{3}$ is larger than a certain critical value, stability of the prediction is lost very quickly. This leads to a fairly sharp upper bound on the estimate of $d_{3}$ for each $n_{l}$. By contrast, stability of the prediction is degraded only gradually if $d_{3}$ is lowered from its optimal value. In this regard, a marked result is that in the case $n_{l}=0$ the exact value of $d_{3}$ is on the verge of or slightly above the upper bound of $d_{3}$ required by stability of the energy. Since US corrections are expected to be the source of IR instability of the energy, we infer that the US corrections are sizable in this case. Oppositely, in the case $n_{l}=5$, the exact value of $d_{3}$ lies slightly below that required by optimal stability of the energy. Hence,

\footnotetext{
${ }^{4}$ Qualitatively the same feature is observed for the heavy quarkonium energy levels [41].
} 


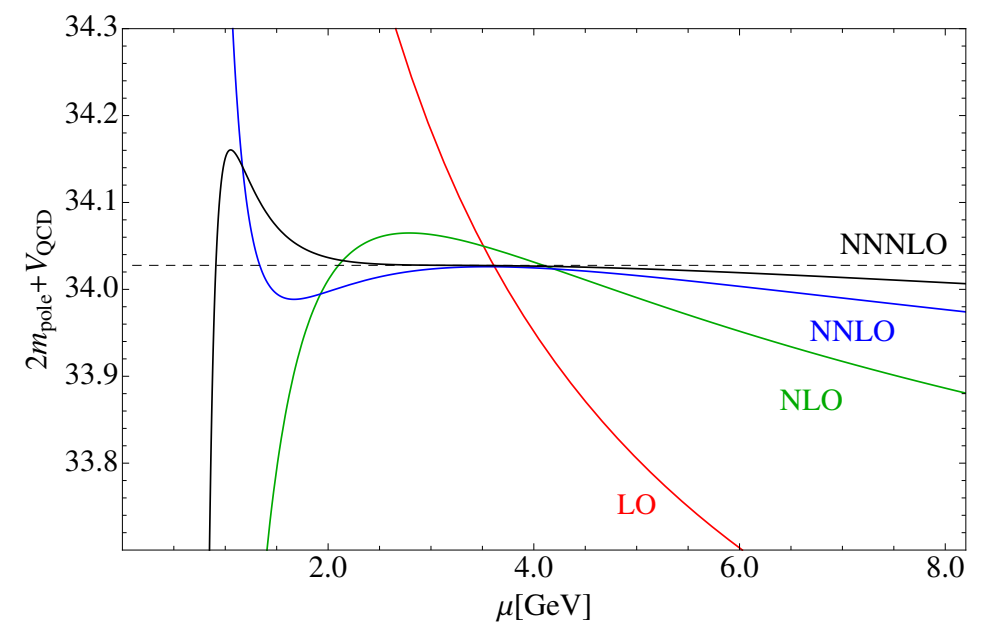

Figure 3. Scale dependences of $2 m_{\text {pole }}+V_{\mathrm{QCD}}(r)$ at different orders of perturbative expansion in the case $n_{l}=0$. Input parameters are $\alpha_{s}(3 \mathrm{GeV})=0.2\left[\alpha_{s}(\mu)\right.$ blows up at $\left.\mu \approx 0.62 \mathrm{GeV}\right], \bar{m}=16 \mathrm{GeV}$ (a large value is chosen to suppress sub-leading renormalons in $m_{\text {pole }}$ ), and $r=0.5 \mathrm{GeV}^{-1}$. The exact value of $d_{3}$ is used. A horizontal line is shown as a guide.

in this case US corrections do not deteriorate perturbative stability in any essential way, and US corrections may well be regarded as "small." Such a dependence of IR stability on $n_{l}$ may result from the fact that the running coupling constant blows up most rapidly for $n_{l}=0$, while the running becomes milder as $n_{l}$ increases. (Note that we consider $n_{l}$ massless quarks.) If an IR catastrophe of perturbative stability should ever occur, it would be expected to appear first in the most rapidly running case. To demonstrate explicitly the level of instability in the case $n_{l}=0$, we show a plot according to the analysis of [4]. Figure 3 shows the scale dependences of $2 m_{\text {pole }}+V_{\mathrm{QCD}}(r)$ at a relatively large $r$, where perturbative stability up to NNLO is close to marginal. The NNNLO line is flatter than the NNLO line in the large $\mu$ region, however, it grows in the small $\mu$ region and starts to show a sign of instability. See [4] for more details of the analysis method.

There is a difficulty in quantifying the size of US corrections more directly. By definition, the US corrections are dependent on the factorization scale $\mu_{f}$, which should satisfy the condition $[10,11]$

$$
\Lambda_{\mathrm{QCD}}, \frac{C_{A} \alpha_{s}}{2 r} \ll \mu_{f} \ll \frac{1}{r},
$$

where $C_{A}=N_{C}=3$ is the Casimir operator for the adjoint representation. Given the different $n_{l}$ dependences of $d_{3}^{\text {est }}[4]$ and $d_{3}^{\text {exact }}[1]$, we confirm that a simple logarithmic dependence of the US corrections on $\mu_{f}$, proportional to $\alpha_{s}^{5} \log \left(\mu_{f} r\right)$, cannot explain the difference, even if we assume a reasonable $n_{l}$ dependence of $\mu_{f}$. This is expected, since except in dimensional regularization, which conceals power-like dependences on the scales, we expect a much stronger dependence $\sim \mu_{f}^{3} r^{2}$ of the US corrections. This dependence should eventually turn into a dependence on the physical US scales, namely $\mu_{f}$ should be replaced by $C_{A} \alpha_{s} /(2 r)$ and $\Lambda_{\mathrm{QCD}}$, where presumably the latter is more dominant at 
larger $r .{ }^{5}$ This requires (at least) an analysis analogous to that of [5] incorporating the $u=3 / 2$ singularity in addition. Furthermore, we would need to separate UV and IR contributions in perturbative expansion systematically, to be able to accurately extract the US contributions $[42,43]$. Such a detailed analysis is beyond the scope of this paper.

Thus, for the case $n_{l}=0$, we are (for the time being) content with the observation that everything is consistent. The renormalon dominance hypothesis can accurately estimate $d_{3}$ by the method of [5]. As mentioned, it is plausible that the renormalon dominance works most accurately in this case. ${ }^{6}$ On the other hand, stability of $2 m_{\text {pole }}+V_{\mathrm{QCD}}(r)$ at IR can in principle be jeopardized by US corrections and, if at all, this is expected to happen for smaller $n_{l}$.

In contrast, for $n_{l}=3,4,5$, the analytic part at $u=1 / 2$ has a larger relative significance, and the central values of the estimates by [5] depart from the exact values; see table 2 and figure 1. We can circumvent this problem in the method of [4], since cancellation of IR dynamics takes place in the analytic part as well, and the contribution of US corrections is expected to be milder than the $n_{l}=0$ case. Thus, we are led to the interpretation as presented in the beginning of this discussion.

On the basis of our understanding up to this point, we reexamine the prediction of the energy level of the (would-be) toponium $1 S$ state, using the NNNLO formula for the $1 S$ energy level [44-46]. We compare with the analysis [47], which examined the $1 S$ energy level calculated in terms of $\bar{m}_{t} \equiv m_{t}^{\overline{\mathrm{MS}}}\left(m_{t}^{\overline{\mathrm{MS}}}\right)$ and in the $\varepsilon$ expansion [48]. The large- $\beta_{0}$ approximation (a crude approximation based on renormalon dominance) was used for estimates of $a_{3}$ and $d_{3} \cdot{ }^{7}$ We replace them by the exact values. The essence of the analysis [47] is to use the renormalon dominance hypothesis for estimating a perturbative error in the top quark mass determination from the energy level of the toponium $1 S$ state. As a result, about $40 \mathrm{MeV}$ for an expected accuracy was predicted for determination of the top quark $\overline{\mathrm{MS}}$ mass.

All the qualitative argument of [47] based on renormalon dominance hypothesis should be valid, since, as we have verified, the renormalon dominance is qualitatively a good approximation. Nevertheless, according to our above understanding, the accuracy of the prediction is expected to improve, since the cancellation of IR dynamics occurs at a deeper level than that of the large- $\beta_{0}$ approximation. In the $t \bar{t}$ system, the leading non-canceled IR contribution from US corrections is expected to be "small" if our understanding is consistent.

Figure 4 compares the scale dependence of the toponium $1 S$ energy by the previous analysis [47] and that using the exact values of $a_{3}$ and $d_{3}$. A marked difference is that the

\footnotetext{
${ }^{5} \mu_{f}$ dependence is canceled in physical observables. Hence, we are ultimately interested in the dependence of physical observables on the physical US scale. At lower orders of perturbative series, only the scale $C_{A} \alpha_{s} /(2 r)$ is visible. As the order is raised, perturbative expansion becomes more sensitive to the $\Lambda_{\mathrm{QCD}}$ scale. The leading dependence of $2 m_{\text {pole }}+V_{\mathrm{QCD}}(r)$ on $\Lambda_{\mathrm{QCD}}$ should appear as $\Lambda_{\mathrm{QCD}}^{3} r^{2}$.

${ }^{6}$ This feature appears to be slightly reinforced for $n_{l}=0$ by a cancellation of the contribution from the $u=-1 \mathrm{UV}$ renormalon and other contributions from the analytic part at $u=1 / 2$; compare tables 2 and 3 .

${ }^{7}$ More accurately, a Padé estimate of $a_{3}$ was used and the prediction of the $1 S$ energy level was shown to be quite close to that of the large- $\beta_{0}$ approximation. Since the difference is minor and irrelevant in our context, we refer only to the large- $\beta_{0}$ approximation.
} 


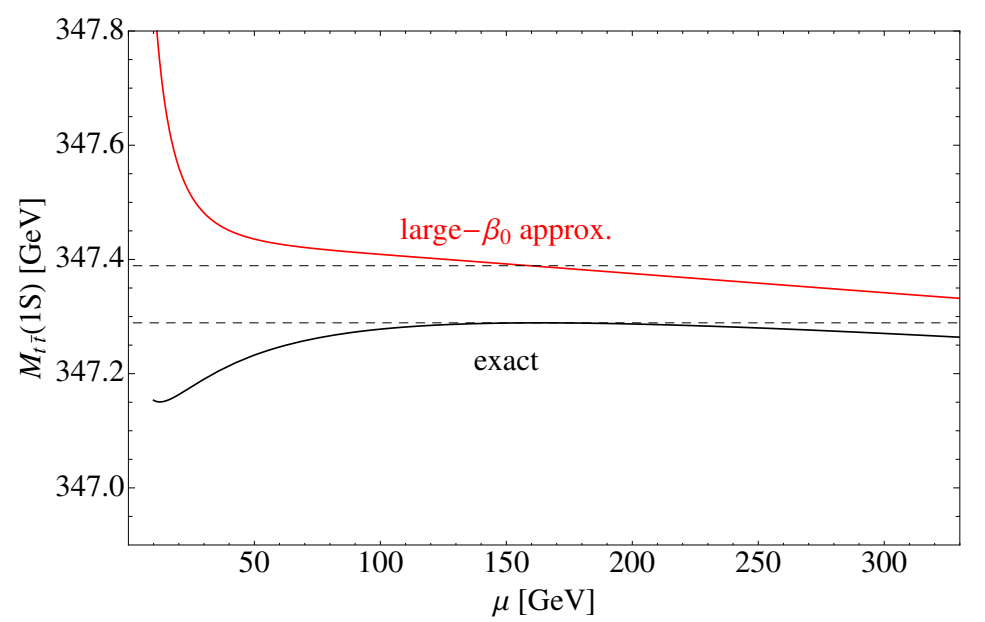

Figure 4. Comparison of scale dependence of the toponium $1 S$ energy at NNNLO from the previous analysis (the large- $\beta_{0}$ approximation [47]) and that using the exact values of $a_{3}$ and $d_{3}$. The input values are $\bar{m}_{t}=165 \mathrm{GeV}, \alpha_{s}\left(M_{Z}\right)=0.1185$, and $n_{l}=5$. Horizontal dashed lines are shown as a guide.

former prediction is much more unstable in the IR region than the latter. This is consistent with our expectation. There also appears a flat region (minimal-sensitivity scale [49]) in the new prediction, which is absent in the former prediction.

We estimate the error of the new prediction. It is natural to use the scale dependence around the minimal-sensitivity scale $(\approx 160 \mathrm{GeV}) .{ }^{8}$ Following the standard prescription we vary the scale by factors $1 / 2$ and 2 . When the scale $\mu$ is varied between 80 and $320 \mathrm{GeV}$, the $1 S$ energy varies by about $20 \mathrm{MeV}$ below and above the minimal-sensitivity scale, respectively. Therefore, the sum of the absolute variations of the $1 S$ energy level is about $40 \mathrm{MeV} .{ }^{9}$ The corresponding variation of the top quark $\overline{\mathrm{MS}}$ mass is almost one half of it, leading to about $20 \mathrm{MeV}$, which we take as an error estimate. Another error estimate may be obtained from the difference between the NNLO prediction at the minimal sensitivity scale (at NNLO) and that at NNNLO, namely the difference between the values of $M_{t \bar{t}}(1 S)$ at the local maxima at NNLO and NNNLO in figure 5. This gives $30 \mathrm{MeV}$ as an uncertainty for the top quark mass. For reference, we show the series expansion in $\varepsilon$ at the minimal sensitivity scale at NNNLO:

$$
M_{t \bar{t}}(1 S)=2 \times(165+7.20+1.22+0.216+0.0077) \mathrm{GeV} \quad \text { for } \quad \mu=162 \mathrm{GeV}
$$

which shows a healthy convergence behavior $\left[\bar{m}_{t}=165 \mathrm{GeV}\right.$ and $\left.\alpha_{s}\left(M_{Z}\right)=0.1185\right]$. Thus, we estimate an error in the top quark $\overline{\mathrm{MS}}$ mass determination from $M_{t \bar{t}}(1 S)$ to be $20-30 \mathrm{MeV}$.

\footnotetext{
${ }^{8}$ From the general argument based on the renormalon dominance hypothesis, the minimal-sensitivity scale is expected to increase as the perturbation order is raised [42]; see figure 5.

${ }^{9}$ This is a factor 2 more conservative estimate than taking the maximal variation of the $1 S$ energy level in this range.
} 
We note that the naive error estimate of order $\Lambda_{\mathrm{QCD}}^{3} /\left(\alpha_{s} m\right)^{2}$ by the uncanceled renormalon at $u=3 / 2$ in [47] is order $3-10 \mathrm{MeV}$, which is still somewhat smaller than the current error estimate. This means that the current perturbative order would not be high enough to be limited by this renormalon uncertainty. Contribution of the renormalon at $u=-1$ in the pole mass is estimated to be a few $\mathrm{MeV}$ or less (see the appendix), while contribution of the $u=+1$ renormalon is estimated naively to be order $\Lambda_{\mathrm{QCD}}^{2} / m \sim 0.5-1.5 \mathrm{MeV}$ (corresponding to $\Lambda_{\mathrm{QCD}} \sim 300-500 \mathrm{MeV}$ ).

In ref. [47] the range of the scale variation was taken differently from the above range, since no minimal-sensitivity scale for the $1 S$ energy exists for that prediction and a different criterion was used. We may check consistency. If we vary the scale in the above range for the previous prediction, we obtain the same error estimate for the top quark $\overline{\mathrm{MS}}$ mass as in [47] (about $40 \mathrm{MeV}$ ).

Thus, we obtained a better possible accuracy of the top quark mass determination at a future linear collider over the previous estimate, which relied only on the renormalon dominance hypothesis before the full computations of $a_{3}$ and $d_{3}$. We consider that it is not a sheer numerical accident but with a reasoning that we obtain a smaller error estimate. Namely, from the general property of QCD a stronger IR cancellation than what is predicted by the renormalon dominance hypothesis follows. This interpretation is supported by a detailed comparison between the estimates of $d_{3}$ for $n_{l}=3,4,5$ from stability of $2 m_{\text {pole }}+V_{\mathrm{QCD}}(r)$ and the estimates by the renormalon dominance, and also by an overall consistent picture drawn in the first part of this paper.

To clarify the current status, we show in figure 5 dependences of the $1 S$ energy level on the current uncertainty of the exact value of $d_{3}$ and on the input value of $\alpha_{s}\left(M_{Z}\right)=$ $0.1185 \pm 0.0006$ [50]. The former induces about $10 \mathrm{MeV}$ variation $(5 \mathrm{MeV}$ for the top quark mass) at the minimal-sensitivity scale, while the latter induces about $90 \mathrm{MeV}(45 \mathrm{MeV}$ for the top mass) variation. Hence, a precise determination of $\alpha_{s}\left(M_{Z}\right)$, of the order of \pm 0.0001 accuracy, is prerequisite to achieve $20-30 \mathrm{MeV}$ accuracy of the top quark mass determination. Prediction of $d_{3}$ with higher precision is also favorable.

For comparison, we perform a similar analysis using the potential subtracted (PS) mass [51] as the input parameter. (The definition of the NNNLO PS mass is given in [44].) Figure 6 shows the scale dependence of the toponium $1 S$ energy level, where we use the PS mass $m_{\mathrm{PS}}\left(\mu_{f, \mathrm{PS}}=20 \mathrm{GeV}\right)=173 \mathrm{GeV}$. To compare with the $\overline{\mathrm{MS}}$ mass, we vary the scale from $80 \mathrm{GeV}$ to $320 \mathrm{GeV}$ and find the variation of the $1 S$ energy level of about $75 \mathrm{MeV}$. (For $50 \mathrm{GeV} \leq \mu \leq 350 \mathrm{GeV}$, the variation is about $100 \mathrm{MeV}$, which is consistent with [52,53].) The uncertainty of $\alpha_{s}\left(M_{Z}\right)$ causes $\pm 8 \mathrm{MeV}$ shift of the NNNLO energy level. ${ }^{10}$ Thus, use of the PS mass leads to a larger scale variation of the perturbative prediction for the $1 S$ energy level than the $\overline{\mathrm{MS}}$ mass. We observe qualitatively different scale dependences between the two schemes by comparing figures 5 and 6 , where this tendency is apparent not only at NNNLO but also at lower orders. Furthermore, we confirm a similar tendency in the scale dependences for other $n_{l}$ 's, where the values of $d_{3}$ vary considerably. We also note

\footnotetext{
${ }^{10}$ The dependence of the PS mass on $\alpha_{S}\left(M_{Z}\right)$ starts from the order $\alpha_{s}^{2}$, which is the reason for a smaller dependence compared to the $\overline{\mathrm{MS}}$ mass.
} 


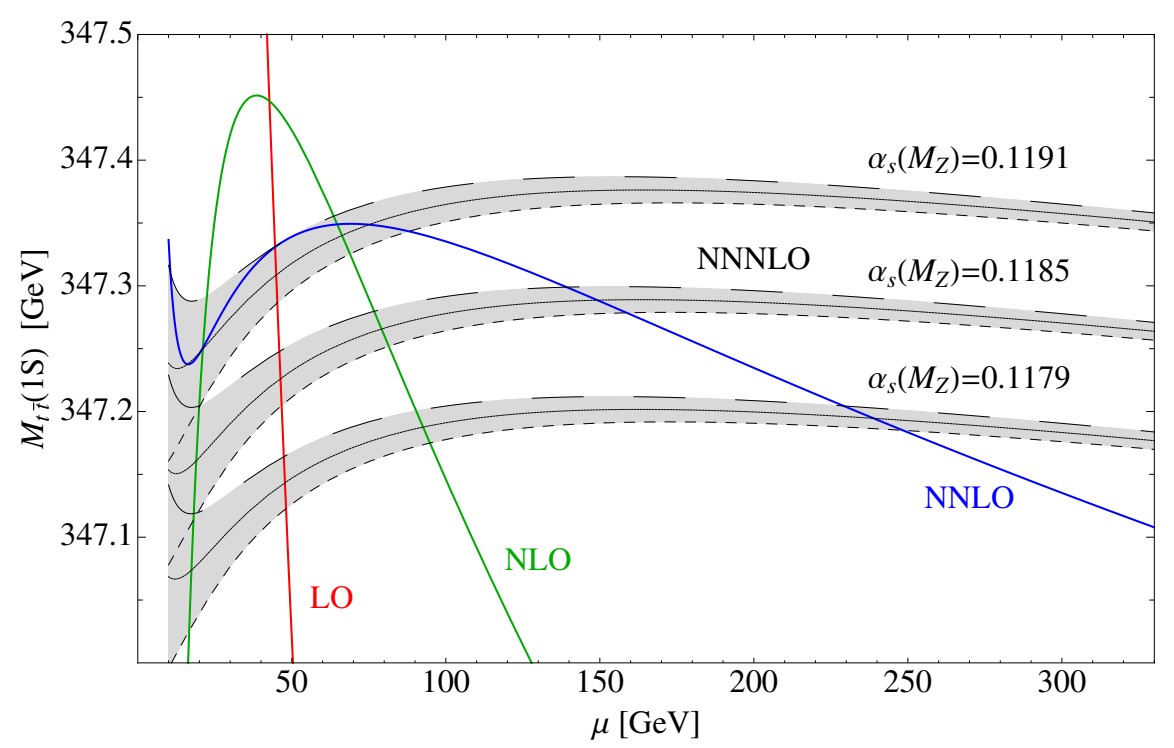

Figure 5. Scale dependence of the toponium $1 S$ energy level. The input $\overline{\mathrm{MS}}$ mass is taken as $\bar{m}_{t}=165 \mathrm{GeV}$. Each band for the NNNLO prediction corresponds to variation of $d_{3}^{\text {exact }}$ inside its error $( \pm 21.5)$, where the upper (lower) line in each band corresponds to the upper (lower) value of $d_{3}^{\text {exact }}$. The different bands correspond to different input values of $\alpha_{s}\left(M_{Z}\right)$. Predictions at lower orders are for $\alpha_{s}\left(M_{Z}\right)=0.1185$.

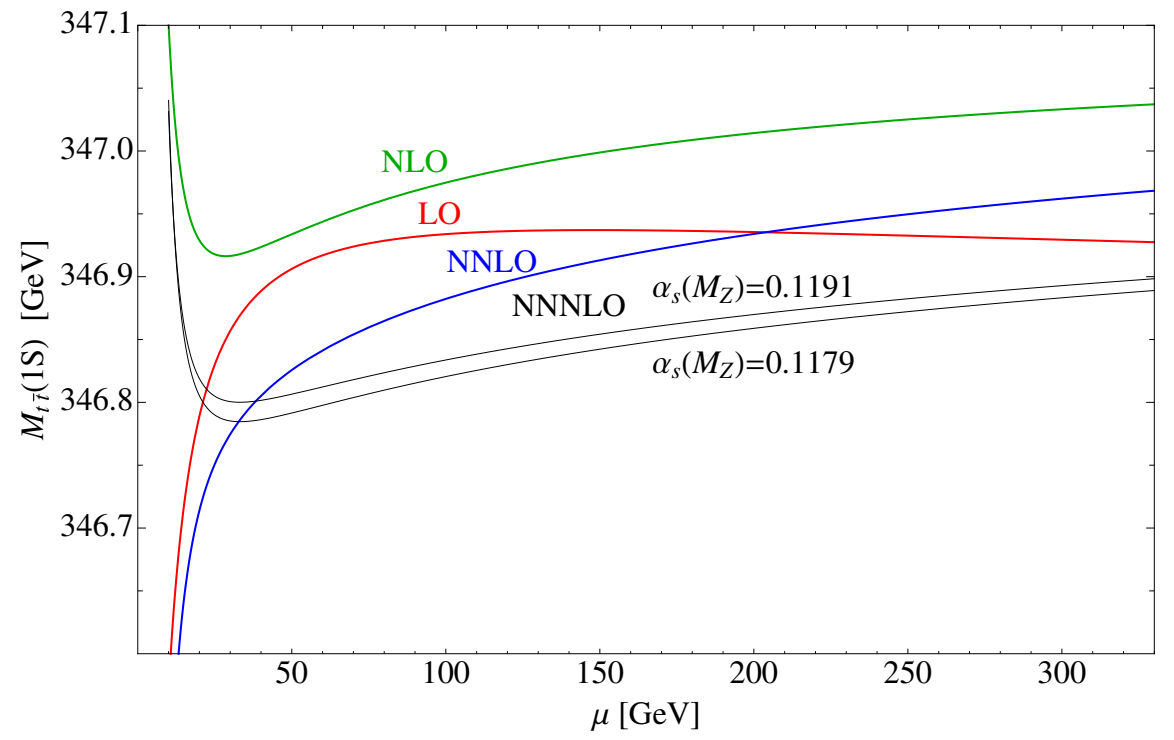

Figure 6. Scale dependence of the $1 S$ energy level in the PS-mass scheme at different orders. The input PS mass is taken as $m_{\mathrm{PS}}\left(\mu_{f, \mathrm{PS}}=20 \mathrm{GeV}\right)=173 \mathrm{GeV}$. Two lines for the NNNLO result correspond to $\alpha_{s}\left(M_{Z}\right)=0.1179$ and 0.1191 . At lower orders $\alpha_{s}\left(M_{Z}\right)=0.1185$ is used.

that the conversion formula between the PS and $\overline{\mathrm{MS}}$ masses induces a scale uncertainty of order $30 \mathrm{MeV}$ for $80 \mathrm{GeV}<\mu<320 \mathrm{GeV}$ provided that $m_{\mathrm{PS}}=173 \mathrm{GeV}$ is an input value. 
Intuitively the difference between using the $\overline{\mathrm{MS}}$ and PS masses may be understood as follows. In the $\overline{\mathrm{MS}}$-mass scheme, the energy of the toponium bound state consists of (i) the $\overline{\mathrm{MS}}$ masses of $t$ and $\bar{t}$, (ii) contributions to the self-energies of $t$ and $\bar{t}$ not renormalized into the $\overline{\mathrm{MS}}$ mass (typically from gluons whose wavelengths are larger than the Compton wavelength of $t, \lambda_{g} \gtrsim 1 / m_{t}$ ), and (iii) the potential energy between $t$ and $\bar{t}$. IR contributions between (ii) and (iii) (typically from $\lambda_{g}$ larger than the bound-state size) get canceled, where the domain of IR cancellation is determined dynamically by the wave function of the bound state $[12,43,54]$.

The composition of the energy of the bound state in the PS-mass scheme is similar, except that the renormalized mass (i) is replaced by the PS mass, which renormalizes the

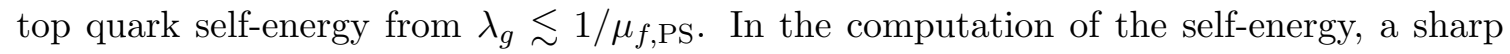
cut-off is introduced in momentum space at the factorization scale $\mu_{f, \mathrm{PS}}$, which is chosen to be of the order of the Bohr scale $\sim \alpha_{s} m_{t}$. The cut-off induces a power dependence of the PS mass on $\mu_{f, \mathrm{PS}}$. Since the $1 / \mu_{f, \mathrm{PS}}$ is close to the bound-state size, the IR cancellation can become incomplete by artificial cut-off effects if $\mu_{f, \mathrm{PS}}$ is too low. Such effects tend to be enhanced, due to the increase of the coupling constant at IR and the power dependence on $\mu_{f, \mathrm{PS}}$.

We may check consistency of this picture, by computing the energy level in the case that $\mu_{f, \mathrm{PS}}$ is taken to be larger than the Bohr scale. ${ }^{11}$ In this case, the behavior of the predictions in the PS-mass scheme is expected to approach qualitatively that of the $\overline{\mathrm{MS}}$ mass scheme, as only shorter-wavelength contributions are renormalized in the PS mass and IR cancellation becomes more complete (artifact of cut-off diminishes). We show in figures $7(\mathrm{a})(\mathrm{b})$ the energy level for $\mu_{f, \mathrm{PS}}=50$ and $80 \mathrm{GeV}$, to be compared with figures 5,6 , and confirm this tendency. (We confirm qualitatively similar behavior for the bottomonium energy level as well.)

Let us discuss other sources of errors. Besides what we have analyzed here, there are many sources of uncertainties, both of theoretical and experimental origins, in the actual top quark mass determination at ILC. Theoretically, these include effects of mixed electroweak and QCD corrections (finite width corrections, non-resonant diagrams, nonfactorizable corrections, etc.), uncertainties in the normalization and shape of the threshold cross section, contributions from higher-spin quarkonium states, method for smooth matching to the high-energy cross section, and so forth. In addition effects of the initial-state radiation and beam energy spread need to be taken into account in a realistic experimental situation for the top quark threshold scan. (See $[55,56]$ for recent simulation studies for the threshold scan at ILC.) Since feasibility of a high precision top quark mass determination can be addressed only by realistic simulation studies incorporating all the above effects, the accuracy we present here is what can be achieved in principle, as a limitation from perturbative QCD. Nevertheless, such a precision is a unique possibility achievable only at a future $e^{+} e^{-}$collider and worth pursuing.

\footnotetext{
${ }^{11}$ In principle this is at odds with the standard counting of $\varepsilon$ in the PS-mass scheme. Furthermore, the approximation of subtracting the IR part of the pole mass by an integral of $-V_{\mathrm{QCD}}(q) / 2$ becomes worse as $\mu_{f, \mathrm{PS}}$ approaches $m_{t}$. Hence, we take the cut-off in the range $\alpha_{s} m_{t}<\mu_{f, \mathrm{PS}}<m_{t}$.
} 


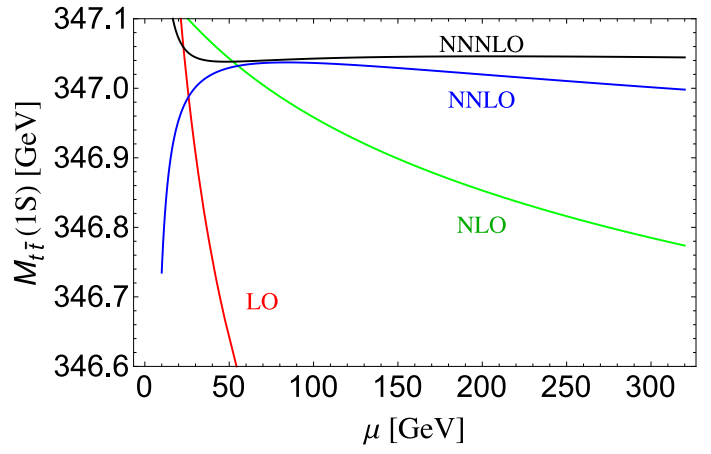

(a)

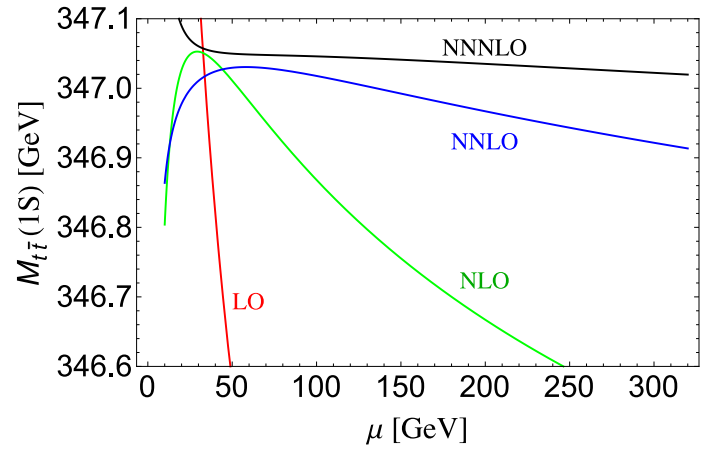

(b)

Figure 7. Scale dependence of the $1 S$ energy level in the PS-mass scheme at different orders in the cases (a) $\mu_{f, \mathrm{PS}}=50 \mathrm{GeV}, m_{\mathrm{PS}}=171.2 \mathrm{GeV}$, and (b) $\mu_{f, \mathrm{PS}}=80 \mathrm{GeV}, m_{\mathrm{PS}}=169.5 \mathrm{GeV}$. The values of the PS mass are chosen such that the lines fit in the same range as in figure 6 .

Note added. After we completed our work, an analysis was reported on the top quark mass determination using the NNNLO $t \bar{t}$ cross section near threshold and using the PS mass $[52,53]$. Their estimate of about $50 \mathrm{MeV}$ accuracy is larger than the estimate presented in this paper $(20-30 \mathrm{MeV})$, which is based only on the uncertainty of the $1 S$ energy level using the $\overline{\mathrm{MS}}$ mass. Currently it remains an open question, in the case that the cross section is computed thoroughly in terms of the $\overline{\mathrm{MS}}$ mass only, whether the latter estimate is increased substantially due to an uncertainty in the shape of the threshold cross section.

\section{Acknowledgments}

The authors are grateful to M. Beneke and M. Steinhauser for useful comments on our manuscript. The authors also thank the editor and the referee for bringing our attention to the $u=1$ renormalon and ref. [36]. The works of Y.K. and Y.S., respectively, were supported in part by Grant-in-Aid for scientific research Nos. 26400255 and 26400238 from MEXT, Japan. The work of G.M. is supported in part by Grant-in-Aid for JSPS Fellows (No. 26-10887).

\section{A UV renormalon}

In this appendix we estimate contributions to the pole- $\overline{\mathrm{MS}}$ mass relation from the UV renormalon at $u=-1$ using the large- $\beta_{0}$ approximation and estimate an uncertainty originating from this renormalon. Using the formula in [33], the contribution to $d_{n}$ [defined in eq. (1)] from the pole at $u=-1$ is given by

$$
\left.d_{n}^{[u=-1]}=e^{-5 / 3} C_{F}(-1)^{n+1}\left(\frac{\beta_{0}}{4}\right)^{n} n ! \quad \text { (large- } \beta_{0} \text { approx. }\right),
$$

where $C_{F}=4 / 3$ is the color factor. In particular the contributions to $d_{3}$ are evaluated explicitly for various $n_{l}$ in table 3 . Comparing these values with the corresponding errors of 


\begin{tabular}{|c|ccccccc|}
\hline$n_{l}$ & 0 & 1 & 2 & 3 & 4 & 5 & 6 \\
\hline$d_{3}^{[u=-1]}$ & 31.4 & 26.1 & 21.3 & 17.2 & 13.7 & 10.6 & 8.1 \\
\hline
\end{tabular}

Table 3. Estimates by the large- $\beta_{0}$ approximation for the contribution of the $u=-1$ UV renormalon to $d_{3}$.

$d_{3}^{\text {est }}[5]$ in table 2 , we find that they are smaller than the errors by factors $4-6$ for $0 \leq n_{l} \leq 4$ and by factors 10-20 for $n_{l}=5,6$. This is consistent, since each error of $d_{3}^{\text {est }}[5]$ is dominated by the contribution from the analytic part at $u=1 / 2$ and $d_{3}^{[u=-1]}$ belongs to the analytic part. It suggests that the $u=-1 \mathrm{UV}$ renormalon is not a dominant component of the contribution from the analytic part (for $n=3$ ).

In the rest of this appendix we estimate the contribution of the $u=-1 \mathrm{UV}$ renormalon to the quarkonium $1 S$ energy level, taking the toponium case $\left(n_{l}=5\right)$ as an example. (The case for the bottomonium is qualitatively similar.)

In the $1 S$ energy level (at the leading-logarithms) only the pole mass contains the $u=$ -1 UV renormalon. In general a UV renormalon induces a factorial growth of perturbative series, as shown in eq. (8) (similarly to an IR renormalon), which breaks convergence of the perturbative series. Nevertheless, since the corresponding singularity in the Borel plane $(u-$ plane) lies along the negative real axis, a definite value can be assigned to the contributions of a UV renormalon by Borel summation. The perturbative series corresponding to a UV renormalon converges up to a certain order $\left(n<n_{*}\right)$ and diverges beyond that order $\left(n>n_{*}\right)$, which is a typical feature of an asymptotic series. In the case of the $u=-1 \mathrm{UV}$ renormalon (the UV renormalon nearest to the origin in the Borel plane), the critical order $n_{*}$ is given by

$$
n_{*} \approx \frac{4 \pi}{\beta_{0} \alpha_{s}\left(\bar{m}_{t}\right)} \approx 15
$$

Therefore, the perturbative series is still converging in our NNNLO calculation. The first several terms of the $u=-1$ contribution (in the large- $\beta_{0}$ approximation) read

$$
\begin{aligned}
M_{t \bar{t}}(1 S)^{[u=-1]} & \equiv 2 \bar{m}_{t}\left[1+\sum_{n=0}^{\infty} d_{n}^{[u=-1]}\left(\frac{\alpha_{s}\left(\bar{m}_{t}\right)}{\pi}\right)^{n+1}\right] \\
& =2 \times[165-1.44+0.096-0.013+0.0025-0.00068+\cdots],
\end{aligned}
$$

for $\mu=\bar{m}_{t}=165 \mathrm{GeV}$ and $\alpha_{s}\left(\bar{m}_{t}\right)=0.109$. According to a standard estimate with an asymptotic series, the error of the prediction is of the order of the last known term. Hence, at NNNLO, we can estimate the error due to the $u=-1$ renormalon to be of order $2.5 \mathrm{MeV}$ for the top quark mass determination.

Alternatively we can estimate the error using the difference between the Borel summed value and the perturbative contribution up to NNNLO:

$$
\begin{aligned}
\delta \bar{m}_{t} & =-\frac{4 C_{F} \bar{m}_{t}}{e^{\frac{5}{3}} \beta_{0}} \int_{0}^{\infty} d u\left[\frac{1}{1+u}-\left(1-u+u^{2}-u^{3}\right)\right] \exp \left[-\frac{4 \pi u}{\beta_{0} \alpha_{S}\left(\bar{m}_{t}\right)}\right] \\
& \approx-0.51 \mathrm{MeV} .
\end{aligned}
$$


It is somewhat smaller than the above estimate. [An error estimate by the $\mathrm{N}^{4} \mathrm{LO}$ term of eq. (11) gives a better estimate.]

From the above examinations, one expects that the contribution of the $u=-1$ renormalon is fairly modest and minor in the error estimate in the determination of the top quark mass, which is performed in the main body of this paper. As long as the perturbative series is converging, the error due to the $u=-1$ renormalon decreases. This is in contrast to the $u=3 / 2$ renormalon, which induces a limitation in achievable accuracy of order $\Lambda_{\mathrm{QCD}}^{3} /\left(\alpha_{s} m_{t}\right)^{2}$. A crude estimate based on the large- $\beta_{0}$ approximation indicates that at NNNLO the error due to the $u=-1$ renormalon is smaller than the error due to the $u=3 / 2$ renormalon.

Open Access. This article is distributed under the terms of the Creative Commons Attribution License (CC-BY 4.0), which permits any use, distribution and reproduction in any medium, provided the original author(s) and source are credited.

\section{References}

[1] P. Marquard, A.V. Smirnov, V.A. Smirnov and M. Steinhauser, Quark Mass Relations to Four-Loop Order in Perturbative QCD, Phys. Rev. Lett. 114 (2015) 142002 [arXiv: 1502.01030] [INSPIRE].

[2] C. Anzai, Y. Kiyo and Y. Sumino, Static QCD potential at three-loop order, Phys. Rev. Lett. 104 (2010) 112003 [arXiv:0911.4335] [INSPIRE].

[3] A.V. Smirnov, V.A. Smirnov and M. Steinhauser, Three-loop static potential, Phys. Rev. Lett. 104 (2010) 112002 [arXiv: 0911.4742] [INSPIRE].

[4] Y. Sumino, Estimate of 4-loop Pole- $\overline{M S}$ Mass Relation from Static QCD Potential, Phys. Lett. B 728 (2014) 73 [arXiv:1309.5436] [INSPIRE].

[5] C. Ayala, G. Cvetič and A. Pineda, The bottom quark mass from the $\Upsilon(1 S)$ system at NNNLO, JHEP 09 (2014) 045 [arXiv:1407.2128] [INSPIRE].

[6] C. Bauer, G.S. Bali and A. Pineda, Compelling Evidence of Renormalons in QCD from High Order Perturbative Expansions, Phys. Rev. Lett. 108 (2012) 242002 [arXiv:1111.3946] [INSPIRE].

[7] T. Appelquist, M. Dine and I.J. Muzinich, The Static Limit of Quantum Chromodynamics, Phys. Rev. D 17 (1978) 2074 [INSPIRE].

[8] M.B. Voloshin, On Dynamics of Heavy Quarks in Nonperturbative QCD Vacuum, Nucl. Phys. B 154 (1979) 365 [INSPIRE].

[9] H. Leutwyler, How to Use Heavy Quarks to Probe the QCD Vacuum, Phys. Lett. B 98 (1981) 447 [INSPIRE].

[10] N. Brambilla, A. Pineda, J. Soto and A. Vairo, Potential NRQCD: An Effective theory for heavy quarkonium, Nucl. Phys. B 566 (2000) 275 [hep-ph/9907240] [INSPIRE].

[11] B.A. Kniehl and A.A. Penin, Ultrasoft effects in heavy quarkonium physics, Nucl. Phys. B 563 (1999) 200 [hep-ph/9907489] [INSPIRE].

[12] N. Brambilla, Y. Sumino and A. Vairo, Quarkonium spectroscopy and perturbative QCD: a new perspective, Phys. Lett. B 513 (2001) 381 [hep-ph/0101305] [INSPIRE]. 
[13] N. Brambilla, Y. Sumino and A. Vairo, Quarkonium spectroscopy and perturbative QCD: massive quark loop effects, Phys. Rev. D 65 (2002) 034001 [hep-ph/0108084] [INSPIRE].

[14] Y. Kiyo and Y. Sumino, Perturbative heavy quarkonium spectrum at next-to-next-to-next-to-leading order, Phys. Lett. B 730 (2014) 76 [arXiv:1309.6571] [INSPIRE].

[15] Y. Sumino, A connection between the perturbative QCD potential and phenomenological potentials, Phys. Rev. D 65 (2002) 054003 [hep-ph/0104259] [INSPIRE].

[16] S. Recksiegel and Y. Sumino, Perturbative QCD potential, renormalon cancellation and phenomenological potentials, Phys. Rev. D 65 (2002) 054018 [hep-ph/0109122] [INSPIRE].

[17] S. Necco and R. Sommer, The $N(f)=0$ heavy quark potential from short to intermediate distances, Nucl. Phys. B 622 (2002) 328 [hep-lat/0108008] [INSPIRE].

[18] A. Pineda, The Static potential: Lattice versus perturbation theory in a renormalon based approach, J. Phys. G 29 (2003) 371 [hep-ph/0208031] [INSPIRE].

[19] S. Recksiegel and Y. Sumino, Comparing the QCD potential in perturbative $Q C D$ and lattice QCD at large distances, Eur. Phys. J. C 31 (2003) 187 [hep-ph/0212389] [INSPIRE].

[20] A. Bazavov, N. Brambilla, X. Garcia i Tormo, P. Petreczky, J. Soto and A. Vairo, Determination of $\alpha_{s}$ from the QCD static energy: An update, Phys. Rev. D 90 (2014) 074038 [arXiv: 1407.8437 ] [INSPIRE].

[21] A. Hoang, P. Ruiz-Femenia and M. Stahlhofen, Renormalization Group Improved Bottom Mass from Upsilon Sum Rules at NNLL Order, JHEP 10 (2012) 188 [arXiv:1209.0450] [INSPIRE].

[22] C. Ayala and G. Cvetič, Calculation of binding energies and masses of quarkonia in analytic QCD models, Phys. Rev. D 87 (2013) 054008 [arXiv: 1210.6117] [InSPIRE].

[23] A.A. Penin and N. Zerf, Bottom Quark Mass from $\Upsilon$ Sum Rules to $\mathcal{O}\left(\alpha_{s}^{3}\right)$, JHEP 04 (2014) 120 [arXiv: 1401.7035] [INSPIRE].

[24] C. Ayala, G. Cvetič and A. Pineda, The bottom quark mass from the $\Upsilon(1 S)$ system at NNNLO, JHEP 09 (2014) 045 [arXiv:1407.2128] [INSPIRE].

[25] M. Beneke, A. Maier, J. Piclum and T. Rauh, The bottom-quark mass from non-relativistic sum rules at NNNLO, Nucl. Phys. B 891 (2015) 42 [arXiv:1411.3132] [INSPIRE].

[26] Quarkonium Working Group collaboration, N. Brambilla et al., Heavy quarkonium physics, hep-ph/0412158 [INSPIRE].

[27] N. Brambilla et al., Heavy quarkonium: progress, puzzles and opportunities, Eur. Phys. J. C 71 (2011) 1534 [arXiv:1010.5827] [INSPIRE].

[28] M. Baak et al., The Electroweak Fit of the Standard Model after the Discovery of a New Boson at the LHC, Eur. Phys. J. C 72 (2012) 2205 [arXiv:1209.2716] [InSPIRE].

[29] M. Ciuchini, E. Franco, S. Mishima and L. Silvestrini, Electroweak Precision Observables, New Physics and the Nature of a 126 GeV Higgs Boson, JHEP 08 (2013) 106 [arXiv: 1306.4644] [INSPIRE].

[30] GFitter Group collaboration, M. Baak et al., The global electroweak fit at NNLO and prospects for the LHC and ILC, Eur. Phys. J. C 74 (2014) 3046 [arXiv:1407.3792] [INSPIRE]. 
[31] G. Degrassi et al., Higgs mass and vacuum stability in the Standard Model at NNLO, JHEP 08 (2012) 098 [arXiv: 1205.6497] [INSPIRE].

[32] D. Buttazzo et al., Investigating the near-criticality of the Higgs boson, JHEP 12 (2013) 089 [arXiv:1307.3536] [INSPIRE].

[33] M. Beneke and V.M. Braun, Naive non-Abelianization and resummation of fermion bubble chains, Phys. Lett. B 348 (1995) 513 [hep-ph/9411229] [INSPIRE].

[34] R. Lee, P. Marquard, A.V. Smirnov, V.A. Smirnov and M. Steinhauser, Four-loop corrections with two closed fermion loops to fermion self energies and the lepton anomalous magnetic moment, JHEP 03 (2013) 162 [arXiv:1301.6481] [INSPIRE].

[35] M. Beneke, More on ambiguities in the pole mass, Phys. Lett. B 344 (1995) 341 [hep-ph/9408380] [INSPIRE].

[36] M. Neubert, Exploring the invisible renormalon: Renormalization of the heavy quark kinetic energy, Phys. Lett. B 393 (1997) 110 [hep-ph/9610471] [INSPIRE].

[37] M. Beneke, Renormalons, Phys. Rept. 317 (1999) 1 [hep-ph/9807443] [InSPIRE].

[38] A. Pineda, Heavy Quarkonium and Nonrelativistic Effective Field Theories, Ph.D. Thesis, Barcelona University, Barcelona Spain (1998).

[39] A.H. Hoang, M.C. Smith, T. Stelzer and S. Willenbrock, Quarkonia and the pole mass, Phys. Rev. D 59 (1999) 114014 [hep-ph/9804227] [INSPIRE].

[40] M. Beneke, A Quark mass definition adequate for threshold problems, Phys. Lett. B 434 (1998) 115 [hep-ph/9804241] [INSPIRE].

[41] Y. Kiyo and Y. Sumino, Perturbative heavy quarkonium spectrum at next-to-next-to-next-to-leading order, Phys. Lett. B 730 (2014) 76 [arXiv:1309.6571] [INSPIRE].

[42] Y. Sumino, Static QCD potential at $r<\Lambda_{Q C D}^{-1}$ : Perturbative expansion and operator-product expansion, Phys. Rev. D 76 (2007) 114009 [hep-ph/0505034] [INSPIRE].

[43] Y. Sumino, Understanding Interquark Force and Quark Masses in Perturbative QCD, arXiv: 1411.7853 [INSPIRE].

[44] M. Beneke, Y. Kiyo and K. Schuller, Third-order Coulomb corrections to the S-wave Green function, energy levels and wave functions at the origin, Nucl. Phys. B 714 (2005) 67 [hep-ph/0501289] [INSPIRE].

[45] A.A. Penin and M. Steinhauser, Heavy quarkonium spectrum at $O\left(\alpha_{s}^{5} m_{q}\right)$ and bottom/top quark mass determination, Phys. Lett. B 538 (2002) 335 [hep-ph/0204290] [INSPIRE].

[46] Y. Kiyo and Y. Sumino, Full Formula for Heavy Quarkonium Energy Levels at Next-to-next-to-next-to-leading Order, Nucl. Phys. B 889 (2014) 156 [arXiv:1408.5590] [INSPIRE].

[47] Y. Kiyo and Y. Sumino, Top mass determination and $O\left(\alpha_{s}^{5} m\right)$ correction to toponium $1 S$ energy level, Phys. Rev. D 67 (2003) 071501 [hep-ph/0211299] [INSPIRE].

[48] A.H. Hoang, Z. Ligeti and A.V. Manohar, B decay and the Upsilon mass, Phys. Rev. Lett. 82 (1999) 277 [hep-ph/9809423] [INSPIRE].

[49] P.M. Stevenson, Optimized Perturbation Theory, Phys. Rev. D 23 (1981) 2916 [InSPIRE]. 
[50] Particle Data Group collaboration, K.A. Olive et al., Review of Particle Physics, Chin. Phys. C 38 (2014) 090001 [inSPIRE].

[51] M. Beneke, A quark mass definition adequate for threshold problems, Phys. Lett. B 434 (1998) 115 [hep-ph/9804241] [INSPIRE].

[52] M. Beneke and M. Steinhauser, Non-relativistic high-energy physics: top production and dark matter annihilation, Nucl. Part. Phys. Proc. 261-262 (2015) 378 [arXiv:1506. 07962].

[53] M. Beneke, Y. Kiyo, P. Marquard, A. Penin, J. Piclum and M. Steinhauser, Next-to-next-to-next-to-leading order QCD prediction for the top anti-top S-wave pair production cross section near threshold in $e^{+} e^{-}$annihilation, arXiv:1506.06864 [INSPIRE].

[54] S. Recksiegel and Y. Sumino, Improved perturbative QCD prediction of the bottomonium spectrum, Phys. Rev. D 67 (2003) 014004 [hep-ph/0207005] [INSPIRE].

[55] M. Martinez and R. Miquel, Multiparameter fits to the $t \bar{t}$ threshold observables at a future $e^{+} e^{-}$linear collider, Eur. Phys. J. C 27 (2003) 49 [hep-ph/0207315] [INSPIRE].

[56] T. Horiguchi et al., Study of top quark pair production near threshold at the ILC, arXiv: 1310.0563 [INSPIRE]. 